(RESEARCH ARTICLE)

\title{
Diagnostic role of interleukin- 6 and quantitative C-reactive protein in suspected early onset neonatal sepsis
}

\author{
Priti Chowdhary *, Ritesh Ranjan and Anita Pandey
}

Subharti Medical College, Meerut, India.

Publication history: Received on 06 October 2020; revised on 18 October 2020; accepted on 24 October 2020

Article DOI: https://doi.org/10.30574/wjarr.2020.8.1.0369

\begin{abstract}
Introduction: Neonatal sepsis is a major cause of morbidity and mortality most remarkable in the third world nations. Early diagnosis and subsequent therapy for the infected infants may play a vital role in lowering such mortality and morbidity rates. Aim: To study the clinical profile of neonatal sepsis in a tertiary care hospital and to correlate the findings with quantitative C-reactive protein (CRP) and Interleukin-6 (IL-6). Settings and Design: A total of 296 neonates admitted in neonatal intensive care unit (NICU) with clinical signs and symptoms suggestive of sepsis were studied. Based on their age the study population was divided into early onset sepsis (EOS): age group less 72 hours and late onset sepsis (LOS): age group more than 72 hours. Also healthy neonates who had no signs and symptoms of sepsis were taken as control for the study. Material and Methods: Blood culture was carried out using BacT/ Alert-3D automated system. Quantitative CRP by nephelometry and IL- 6 by ELISA was done in all culture positive cases and controls. Correlation of detection of cases of sepsis by quantitative CRP and IL-6 with blood culture was carried out. Statistical analysis: Statistical parameters such as sensitivity, specificity, predictive values, accuracy and significance levels were calculated Results: In EOS the sensitivity and negative predictive value (NPV) of IL-6 was $62.32 \%$ and $33.33 \%$ respectively as compared to sensitivity of $27.5 \%$ and NPV of $26.47 \%$ of CRP. Conclusions: IL- 6 is a good marker for early onset sepsis than CRP detecting a greater number of sepsis cases.
\end{abstract}

Keywords: Early onset sepsis; late onset sepsis; IL-6, C - reactive protein

\section{Introduction}

Neonatal sepsis is a major cause of morbidity and mortality most remarkable in the third world nations.[1] Neonatal sepsis presents with varied symptoms and signs. Early diagnosis and subsequent therapy for the infected infants or those with a higher risk may play a vital role in lowering such mortality and morbidity rates.[1]

Blood culture is the most common method used for the diagnosis of neonatal sepsis. However, it has certain disadvantages, especially diagnosis often being retrospective. The outcome of sepsis in neonates is directly related to the early management of the disease, and hence prompt use of proper antibiotic therapy is absolutely essential.[2] However, in order to prevent microbial resistance induced by unnecessary administration of empiric therapies and avoiding overwhelmed hospital admissions, a presumptive diagnosis should be secured based on the laboratory tests with higher diagnostic value. Thus, there is a need for an alternative method of diagnosis of Neonatal sepsis which is rapid, less cumbersome and accurate. This has prompted us to carry out the present study with the aim to evaluate the role of quantitative C-reactive protein (CRP) and Interleukin -6 (IL-6) in cases of Neonatal sepsis.

\footnotetext{
${ }^{*}$ Corresponding author: Dr Priti Chowdhary,

Microbiology, Subharti Medical College, Meerut, India.
} 


\section{Material and methods}

A total of 296 clinically suspected cases of neonatal septicaemia admitted in neonatal intensive care unit (NICU) of a tertiary care hospital were studied over a period of one year. Neonates with clinical signs and symptoms suggestive of sepsis were included in study. Infants who were already on antibiotics were excluded from the study. Neonates were divided into two groups on the basis of their age. Early onset sepsis (EOS) i.e. neonates of age group less than 72 hours and late onset sepsis (LOS) i.e. neonates of age group more than 72 hours. The study was approved by the ethical committee of the institution, and written informed consent was obtained from the parents of the neonates before collection of clinical samples.

Blood samples were collected for blood culture and serology before starting treatment. The blood sample inoculated in the BacT/ Alert-3D paediatric culture bottle was incubated in an automated microbial detection system (bioMerieux) for up to 5 days at 370C. Any growth indicated by flagging was sub cultured on blood agar and MacConkeys agar plates [Hi-Media Pvt. Ltd., Mumbai]. The bacteria isolated were identified as per standard bacteriological technique.[3]

Samples were centrifuged within 30 minutes of collection for separation of serum for performing quantitative CRP by Nephelometry (NEPHSTAR, quantitative CRP Kit). Remaining serum was stored at -200C before analysis for determination of IL-6. IL-6 measurement was performed by commercial ELISA kit (DIACLONE, ELISA), as per the instruction given in kit literature. We defined the cut off values for both CRP and IL-6. A C-reactive protein value of less than $5 \mathrm{mg} / \mathrm{L}$ was taken as negative. [4, 5] We did literature search for optimum cut off value of IL-6. An IL-6 value of less than $20 \mathrm{pg} / \mathrm{ml}$ was taken as negative. [6-10]

We are comparing the sensitivities of two diagnostic procedures IL6 and CRP, where sensitivity is defined as "How often a test correctly diagnoses a positive result for the patients who have the disease i.e. true positives". A highly sensitive test will correctly exclude patients who do not have the disease (true negative) but will not generate many false positive results. Therefore, a test that has a higher sensitivity is preferred. Increased sensitivity will lead to decreased specificity (ability to correctly identify true negatives) and vice-versa.

In this study we are considering sensitivity as the characteristic of interest. Since both diagnostic tests are performed on same group of patients, it results in paired data and the appropriate statistical test in such a situation is McNemar's Chi-square test. This will specify if the sensitivities of the two tests are statistically similar or not. The statistical software used here is SPSS version 16.0

\section{Results and discussion}

A total of $135 / 296$ (45.5\%) cases were blood culture positive. Pure growth of bacteria and pure growth of Candida species were isolated from 128/296 (43.24\%) cases. Mixed growth of bacteria plus yeast was isolated from 6/296 (0.02 $\%$ ) of cases, respectively. Neonatal sepsis was defined as the presence of a positive blood culture containing pure growth of bacteria or Candida with supportive clinical features. A total of 69/128 (53.90\%) culture positive cases of sepsis, were in the age group of $<72$ hours, i.e. early onset sepsis (EOS) and 59/128 (46.09\%) cases were in the age group $>72$ hours-28 days i.e. late onset sepsis (LOS).

\subsection{Clinical presentation and risk factor}

We retrospectively correlated our results of Neonatal sepsis with the clinical presentation of neonates and associated risk factors. It was observed that lethargy and feed intolerance were the most common clinical presentation present in almost $100 \%$ of cases of EOS and LOS, followed by respiratory distress in $78.4 \%$ and $57.7 \%$ of cases of EOS and LOS cases respectively. Bleeding tendency (9.8\% \& 17.9\%), convulsions $(15.6 \% \& 32.05 \%)$ and poor perfusion $(23.53 \&$ $41 \%$ ) were the other less common clinical presentations in EOS and LOS respectively.

Low birth weight (74.51\% \& 66.67\%) and prematurity $(74.51 \%$ \& 66.67\%) were the most common risk factors present in both EOS and LOS cases. Prolonged intravenous antibiotics (5.88\% \& 10.26\%), ventilator support (9.80\% \& 15.39\%), prolonged hyperalimentation $(11.76 \% \& 10.26 \%)$ and prolonged central venous line (5.88\% \& $10.26 \%)$ were other less common risk factors observed in EOS and LOS cases respectively. 


\subsection{Estimation of IL-6 and CRP}

IL-6 and CRP estimation was done in 129 culture positive cases belonging to both EOS and LOS group. IL-6 and CRP estimation was also done in few randomly chosen healthy cases both from EOS and LOS groups whose blood culture was negative. Positive and negative values were determined according to cut off values defined earlier.

\subsection{IL-6}

In cases of EOS, IL6 was positive in 43/69 (62.31\%) cases which were also positive by blood culture with a mean value of $173.34 \mathrm{pg} / \mathrm{ml}$. IL6 was positive in $8 / 19$ (42.10\%) control cases which were negative on blood culture and had no sign and symptoms of sepsis (mean value of $66.17 \mathrm{pg} / \mathrm{ml}$ ). However, in cases of LOS, IL-6 was positive in 28/59(47.45\%) cases which were positive by blood culture (mean value of $74.49 \mathrm{pg} / \mathrm{ml}$ ) and $7 / 26(26.92 \%$ ) healthy neonates which were negative on blood culture (mean value of $7.52 \mathrm{pg} / \mathrm{ml}$ ).

\subsection{Quantitative CRP}

CRP was positive in 19/69 (27.53\%) cases of EOS which were positive by blood culture (mean value $17.04 \mathrm{mg} / \mathrm{l}$ ) and $01 / 19(5.26 \%)$ healthy neonates which were negative on blood culture (mean value $04.86 \mathrm{mg} / \mathrm{l}$ ). However, in cases of LOS, CRP was positive in 29/59(49.15\%) cases which were positive by blood culture (mean value 16.90 mg/l) and 7/26 $(26.92 \%)$ healthy neonates which were negative on blood culture (mean value of $0.99 \mathrm{mg} / \mathrm{l}$ ).

\subsection{Comparison of IL-6 and quantitative CRP with blood culture}

\subsubsection{Comparison between IL6 and CRP with blood culture}

Table 1 Comparison of IL-6 and CRP with blood culture positive and blood culture negative cases in EOS

\begin{tabular}{|l|l|l|l|}
\hline \multirow{4}{*}{ IL6 } & \multicolumn{2}{|l|}{ CRP } & \multicolumn{2}{l|}{} \\
\cline { 2 - 4 } & & CRP Positive & CRP Negative \\
\cline { 2 - 4 } & IL6 Positive & 13 & 30 \\
\cline { 2 - 4 } & IL6 Negative & 6 & 20 \\
\hline
\end{tabular}

Considering the sensitivities of the two diagnostic tests as our statistic of interest. The estimates of sensitivities of IL6 and CRP are $s 1=62.32 \%$ and $s 2=27.5 \%$ respectively. IL6 and CRP are applied to a given set of individuals, some with the disease (by the gold standard) and some without the disease.

The table above gives a 2 X2 table for the 69 blood culture positive patients. The appropriate statistical test in such a situation is McNemar's test.The patients with both tests positive and those with both tests negative do not distinguish between the two diagnostic procedures. The only information for distinguishing between IL6 and CRP comes from the patients who test positive by IL6 and negative by CRP or negative by IL6 and positive by CRP.

We test the following null hypothesis:

H0: The sensitivities of IL6 and CRP are equal, s1=s2.

Against the alternative H1: The sensitivity of IL6 is statistically higher than that of CRP (One tailed test)

Table 2 Profile of comparison of IL-6 and CRP with blood culture positive and blood culture negative cases in LOS

\begin{tabular}{|l|l|l|l|}
\hline \multirow{4}{*}{ IL6 } & \multicolumn{2}{|l|}{ CRP } \\
\cline { 2 - 4 } & & CRP Positive & CRP Negative \\
\cline { 2 - 4 } & IL6 Positive & 13 & 15 \\
\cline { 2 - 4 } & IL6 Negative & 16 & 15 \\
\hline
\end{tabular}

On performing the McNemar's test with the above data, the value of test statistic is 14.7 and the exact pvalue for a one tailed test is 0.0001 .On comparing this $p$ value to a standard threshold of $p=0.05$, we find that pvalue $<0.05$ and hence 
we reject the null hypothesis and conclude that sensitivity of IL6 is statistically higher than that of CRP. Hence, in early onset sepsis one should prefer IL6 to CRP in detecting sepsis cases.

From table 2 we see that sensitivities of IL6 and CRP are $47.46 \%$ and $49.15 \%$ respectively but the specificities are same. But we still need to check if the two sensitivities are statistically equal or not.

For this we set up the null hypothesis:

H0: The two sensitivities are statistically equal, s1=s2.

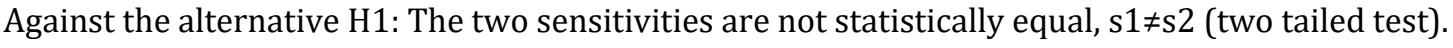

On performing McNemar's test for the above data, we find that the value of test statistic is 0.000 and the exact $p$ value for a two tailed test is 1.000 which is greater than the standard threshold of $p=0.05$ and thus we fail to reject the null hypothesis at $5 \%$ level of significance and conclude that the sensitivity of IL6 and CRP are statistically equal and there is no reason to prefer one to the other in case of late onset sepsis for disgnosis of sepsis cases.

\subsection{Statistical parameters}

Statistical Parameters showing the Sensitivity, Specificity, Positive and Negative predictive value and Accuracy of IL6 and CRP estimation in relation to Blood culture in both EOS and LOS was calculated [Table 2].

Table 3 Statistical Parameters showing the Sensitivity, Specificity, Positive and Negative predictive value and Accuracy of IL6 and CRP estimation in relation to Blood culture in both EOS and LOS.

\begin{tabular}{|c|c|c|c|c|c|c|c|c|}
\hline \multirow[t]{2}{*}{ Parameter } & \multicolumn{4}{|l|}{ EOS } & \multicolumn{4}{|l|}{ LOS } \\
\hline & IL6 & $95 \% \mathrm{CI}$ & CRP & $95 \% \mathrm{CI}$ & IL6 & $95 \% \mathrm{CI}$ & CRP & $95 \% \mathrm{CI}$ \\
\hline Prevalence & $78.41 \%$ & $\begin{array}{l}(68.35 \%, \\
86.47 \%)\end{array}$ & $78.4 \%$ & $\begin{array}{l}(68.35 \%, \\
86.47 \%)\end{array}$ & $69.41 \%$ & $\begin{array}{l}\text { (58.47\%, } \\
78.95 \%\end{array}$ & $69.41 \%$ & $\begin{array}{l}\text { (58.47\%, } \\
78.95 \%\end{array}$ \\
\hline Sensitivity & $62.32 \%$ & $\begin{array}{l}\text { (49.83\%, } \\
73.71 \%\end{array}$ & $27.5 \%$ & $\begin{array}{l}(17.46 \%, \\
39.62 \%)\end{array}$ & $47.46 \%$ & $\begin{array}{l}(34.30 \%, \\
60.88 \%\end{array}$ & $49.15 \%$ & $\begin{array}{l}(35.89 \%, \\
62.50 \%)\end{array}$ \\
\hline Specificity & $68.42 \%$ & $\begin{array}{l}(43.45 \%, \\
87.42 \%\end{array}$ & $94.7 \%$ & $\begin{array}{l}(73.97 \%, \\
99.87 \%)\end{array}$ & $73.08 \%$ & $\begin{array}{l}(52.21 \%, \\
88.43 \%\end{array}$ & $73.08 \%$ & $\begin{array}{l}(52.21 \%, \\
88.43 \%)\end{array}$ \\
\hline $\begin{array}{l}\text { Positive } \\
\text { Predictive } \\
\text { Value }\end{array}$ & $87.76 \%$ & $\begin{array}{l}\text { (78.29\%, } \\
93.44 \%\end{array}$ & $95.00 \%$ & $\begin{array}{l}(73.08 \%, \\
99.25 \%)\end{array}$ & $80.0 \%$ & $\begin{array}{l}(66.78 \%, \\
88.84 \%)\end{array}$ & $80.56 \%$ & $\begin{array}{l}(67.63 \% \text {, } \\
89.15 \%)\end{array}$ \\
\hline $\begin{array}{l}\text { Negative } \\
\text { Predictive } \\
\text { Value }\end{array}$ & $33.33 \%$ & $\begin{array}{l}(24.53 \%, \\
43.47 \%\end{array}$ & $26.47 \%$ & $\begin{array}{l}(23.12 \%, \\
30.12 \%)\end{array}$ & $38.00 \%$ & $\begin{array}{l}(30.45 \%, \\
46.18 \%)\end{array}$ & $38.78 \%$ & $\begin{array}{l}(31.02 \%, \\
47.15 \%)\end{array}$ \\
\hline Accuracy & $63.64 \%$ & $\begin{array}{l}\text { (52.69\%, } \\
73.63 \%\end{array}$ & $42.05 \%$ & $\begin{array}{l}(31.60 \%, \\
53.05 \%)\end{array}$ & $55.29 \%$ & $\begin{array}{l}(44.11 \%, \\
66.09 \%)\end{array}$ & $56.47 \%$ & $\begin{array}{l}(45.28 \%, \\
67.20 \%)\end{array}$ \\
\hline
\end{tabular}

\subsection{Clinical Outcome}

On comparing the clinical outcome of sepsis cases not much difference was observed between the two groups (EOS and LOS). We observed that the survival rate was slightly higher in cases of LOS (93\% survival) as compared to EOS ( $90 \%$ survival). However, the actual clinical outcome could not be followed up as many neonates were transferred out from the NICU, discharged or taken against medical advice. 


\section{Discussion}

The blood culture positivity rate in cases of neonatal sepsis in the present study was $45.5 \%$, which is comparable to other studies from India. Desai et al. [11] and Jain et al. [12] reported culture positivity rate of $46.20 \%$ and $56.6 \%$ respectively in their studies. Cuture positivity rate varying from $25 \%$ to $60 \%$; have been reported in different Indian studies. [13-16]. Pure growth of bacteria was isolated from $59.44 \%$ cases out of total culture positive samples in our study. Similarly, a high blood culture-positivity rate in neonatal septicaemia had been reported by various Indian authors. $[12,14]$

In the present study lethargy and feed intolerance were the most common clinical presentation observed in almost all our cases in both the groups (EOS \& LOS), followed by respiratory distress. Bleeding tendency, convulsions and poor perfusion were the other less common clinical presentations observed. Similarly, according to a recent study by De $e t$ al [17] the major symptoms of neonatal sepsis were lethargy and poor feeding. Christian et al [18] have reported that bacterial infection is another possible cause of neonatal respiratory distress.

Low birth weight (74\%) and prematurity (66\%) were the most common risk factor identified in both EOS and LOS patients. However, prolonged IV antibiotics, ventilator support, prolonged hyperalimentation and prolonged central venous line were other less common risk factors identified in this study and were associated more with LOS than EOS patients. Similarly, De et al. [17] also reported low birth weight and prematurity as major foetal risk factors. In another study Stoll et al. [19] published their results as rate of infection was inversely related to birth weight and gestational age. Complications of prematurity associated with an increased rate of late-onset sepsis included patent ductus arteriosus, prolonged ventilation, prolonged intravascular access, bronchopulmonary dysplasia, and necrotizing enterocolitis. Infants who developed LOS had a significantly prolonged hospital stay (mean length of stay: 79 Vs 60 days). The mortality rates were significantly higher than those who were not infected (18\% Vs $7 \%$ ), especially if they were infected with Gram-negative organisms (36\%) or fungi (32\%). Wai et al [20] also concluded that neonatal sepsis contributes to great mortality and morbidity among very-low-birth-weight (VLBW) infants. Unlike term infants, Gramnegative organism and $E$. coli were the leading pathogen of EOS among VLBW infants. Judicious and timely use of antibiotic therapy is crucial in the care of VLBW infants. Dankner et al. [21] concluded that major risk factor for neonatal sepsis includes prolonged hospitalization, the presence of central venous catheters, and the use of intravenous fat emulsions. Similar risk factors were identified in our study.

In the present study we calculated the statistical parameters that is the sensitivity, specificity, positive predictive value (PPV), negative predictive value (NPV) and accuracy of IL6 and CRP estimation in relation to blood culture positivity in both the EOS and LOS patients. [Table 3]. Celik et al. [10] have reported the sensitivity, specificity, PPV, and NPV of IL6 , when compared to blood culture for neonatal sepsis to be $72 \%, 84 \%, 95 \%$, and $42 \%$, respectively when the cut-off value in the diagnosis of neonatal sepsis was taken as $24.65 \mathrm{pg} / \mathrm{ml}$ and for CRP the sensitivity, specificity, PPV, and NPV was $67 \%, 97 \%, 99 \%$, and 39\%, respectively when compared to blood culture when the cut off value was $4.82 \mathrm{mg} / \mathrm{l}$. Different workers have worked with different cut off levels of IL6 for EOS. To refer to a few reported cases: Berner et al. [22] reported sensitivity, specificity, PPV and NPV as 87\%, 93\%, 76\% and 97\% at cut off level at $100 \mathrm{pg} / \mathrm{ml}$. Dollner et al.[23] had cut off level $33 \mathrm{pg} / \mathrm{ml}$ and got sensitivity and specificity as $84 \%$ and $70 \%$ respectively. In the same year Krueger et al. [24] had cut off level as $80 \mathrm{pg} / \mathrm{ml}$ and reported the sensitivity and specificity as $96 \%$ and $95 \%$ respectively. Santana et al. [25] also had cut off level of $100.8 \mathrm{pg} / \mathrm{ml}$ but reported sensitivity of 50\% and specificity, PPV and NPV of $87 \%, 31 \%$ and $66 \%$ respectively. In another study Tasci et al. [26] reported sensitivity, specificity, PPV and NPV of IL6 as $100 \%, 81 \%, 37 \%$ and $100 \%$ with cut off level at $39 \mathrm{pg} / \mathrm{ml}$.

In the present study the sensitivity of CRP when compared to blood culture positivity was $27.5 \%$ in cases of EOS. Similar finding has been reported by Bhat et al. [27] in their study. In another study the sensitivity, specificity, PPV and NPV of CRP as compared to blood culture positivity has been reported as 50\%, 97\%, 67\% and 94\% respectively with the cutoff value of $5 \mathrm{mg} / \mathrm{L}$. [28] Døllner et al. [23] found that CRP levels were undetectable in nearly all of the neonates, both in infectious and control groups. Santana et al. [25] using a more sensitive method allowing a detection limit of $1 \mathrm{mg} / \mathrm{dL}$, found that the cord blood CRP levels were low $(0.076-0.63 \mathrm{mg} / \mathrm{dL}$ ) and of no significant difference among infectious, non-infectious and healthy groups.

The present study therefore highlights that on comparative evaluation of biomarkers it was found that IL-6 was a more sensitive biomarker and rises early in Neonatal sepsis compared to CRP. CRP has been proved as a "specific" but "late" marker of neonatal infection [23]. Both biomarkers showed comparable results in cases of LOS. However, to exactly pinpoint the biomarkers, a greater number of patients of neonatal sepsis including various groups (fungal, anaerobic etc.) needs to be studied in future. Moreover, as IL-6 is an early indicator its level needs to be monitored on hourly basis to come to a conclusion. These are the few limitations of our study. 
On calculation of the mean value of IL- 6 and CRP, the present study highlighted that, the mean positive value of IL-6 was higher in patients with positive blood culture $(173.34 \mathrm{pg} / \mathrm{ml})$ in EOS as compared to LOS where the mean value of IL-6 in culture positive cases was $74.49 \mathrm{pg} / \mathrm{ml}$.

In the present study we could see that the mean value of both IL-6 and CRP were definitely higher in blood culture positive cases of Neonatal sepsis as compared to blood culture negative cases.

Evidences confirm that IL-6 levels increase rapidly after exposure to bacterial components. Kuster et al.[8] reported median value of IL-6, $5.6 \mathrm{pg} / \mathrm{ml}$ in no sepsis group and $89.7 \mathrm{pg} / \mathrm{ml}$ in proven sepsis group. In a recent study Arani et al. [28] reported the average level of IL-6 in the group admitted with signs and symptoms of sepsis and a positive blood culture was $1545.65 \mathrm{pg} / \mathrm{ml}$ and in the group admitted with signs and symptoms of sepsis and a negative blood culture was $14.79 \mathrm{pg} / \mathrm{ml}$, while in the control group, it was $11.04 \mathrm{pg} / \mathrm{ml}$. In cases of EOS, the mean value of CRP in culture positive and negative cases was $17.04 \mathrm{mg} / \mathrm{l}$ and $04.86 \mathrm{mg} / \mathrm{l}$ respectively. Similarly in LOS, the mean value of CRP was $16.90 \mathrm{mg} / \mathrm{l}$ and $0.99 \mathrm{mg} / \mathrm{l}$. in culture positive \& negative cases respectively. Chiesa et al. [7] in their study found geometric mean CRP values were significantly higher in the infected than in the uninfected neonates at birth [12.0 Vs $3.3 \mathrm{mg} / \mathrm{l}]$, at $24 \mathrm{~h}[37.0 \mathrm{Vs} 4.7 \mathrm{mg} / \mathrm{l}]$, and at $48 \mathrm{~h}$ of age [33.5 Vs $4.5 \mathrm{mg} / \mathrm{l}]$.

IL-6 is a cytokine of the early host response to infection, preceding the increase of CRP and after the release of TNF- $\alpha$. It is synthesized by mononuclear phagocytes, endothelial cells, fibroblasts, decidua, chorion, amnion and trophoblastic cells shortly after stimulus of microbial products.[26] In vivo experiments have shown that the release, peak response and declination to baseline of IL- 6 was observed at 1-2 hours, 3 hours and 8 hours, respectively, after lipopolysaccharide (LPS) injection. The half-life time is considered to be around 100 minutes and its circulating concentration can drop precipitously following antimicrobial treatment [5].

Due to lack of adequate facility of virology laboratory, we could not include viral infection sepsis cases in our study. This was the limitation of our study.

\section{Conclusion}

The present study highlights that IL-6 is a good marker for early onset neonatal sepsis detecting a greater number of cases than CRP. So, Il 6 should be introduced in routine diagnosis of neonatal sepsis. It will prevent the delay in treatment while waiting for blood culture report. In this way it will reduce the mortality and morbidity due to early onset neonatal sepsis.

\section{Compliance with ethical standards}

\section{Acknowledgments}

Dr Molly Madan, Professor and Head, Department of Microbiology, Subharti Medical College, Meerut-250005.

\section{Disclosure of conflict of interest}

There was no conflict of interest.

\section{Statement of ethical approval}

Ethical approval was taken from institutional ethical committee.

\section{Statement of informed consent}

Informed consent was obtained from all individual participants included in the study.

\section{References}

[1] Abdollah A, Shoar S, Nayyeri F, and Shariat M. Diagnostic Value of Simultaneous Measurement of Procalcitonin, Interleukin 6 and Hs CRP in Prediction of Early Onset Nenatal Sepsis. Mediterr J Hematol Infect Dis. V.4(1);2012>PMC 3375671.

[2] Fioretto JR, Martin JG, Kurokawa CS, Carpi MF, Bonatto RC, Ricchetti SM, et al. Interleukin-6 and procalcitonin in children with sepsis and septic shock. Cytokine. 2008 Aug; 43(2):160-4. 
[3] Collee JG, Miles RS, Watt B. Tests for the Identification of Bacteria. In Collee JG, Fraser AG, Mermion BP, Simmons A. (Ed.), Mackie \& McCartney Practical Medical Microbiology, 14/e, 131-150.

[4] Urdal P, Borch SM, Landass S, Krutness MB, Gogstad GD, Hjordahi P. Rapid Immunometric Measurement of CReactive Protein in Whole Blood. Clin. Chem. 1992; 38:580-584.

[5] Morley JJ, Kushner I. "Serum C-Reactive Protein Levels in Disease. Ann NY Acad Sci 1982; 389:406-18.

[6] Küster $\mathrm{H}$, Weiss $M$, Willeitner AE, et al. Interleukin-1 receptor antagonist and interleukin-6 for early diagnosis of neonatal sepsis 2 days before clinical manifestation. Lancet 1998; 352: 1271-77.

[7] Chiesa C, Pellegrini G, Panero A, Osborn JF, Signore F, Assumma M, and Pacifico L. C-Reactive Protein, Interleukin6, and Procalcitonin in the Immediate Postnatal Period: Influence of Illness Severity, Risk Status, Antenatal and Perinatal Complications, and Infection. Clinical Chemistry 49:1; 60-68 2003.

[8] Esra Önal E, Kitapçi F, Dilmen U, Adam B. Interleukin-6 concentrations in neonatal sepsis. THE LANCET • Vol 353 - January 16, 1999; 239-40.

[9] Prinsen JH, Baranski E, Posch H, Tober K, Gerstmeyer A. Interleukin-6 as diagnostic marker for neonatal sepsis: determination of Access IL-6 cutoff for newborns. Clin Lab. 2008; 54(5-6):179-83.

[10] Celik IH, Demirel FG, Uras N, Oguz SS, Erdeve O, Biyikli Z, Dilmen U. What are the cut-off levels for IL-6 and CRP in neonatal sepsis? J Clin Lab Anal. 2010; 24(6):407-12. doi: 10.1002/jcla.20420.

[11] Desai KJ, Haider S, Malek S. Neonatal Septicemia: Bacterial Isolates \& Their Antibiotics Susceptibility Patterns. NJIRM 2010; Vol. 1(3). July-Sept. 12-15.

[12] Jain NK, Jain VM, Maheshwari S. Clinical Profile of Neonatal sepsis. Kathmandu Univ Med J 2003; 1(2):117-20.

[13] Neonatal morbidity and mortality; Report of the National Neonatal Perinatal Database. Indian Paediatr 1997; 34(11):1039-42.

[14] Sharma PP, Halder D, Dutta AK, Dutta R, Bhatnagar S Bali A et al. Bacteriological profile of neonatal septicaemia. Indian Paediatr.1987; 24:1011-7.

[15] Mathur NB. Neonatal sepsis, Indian Paediatr 1996; 33:663-74.

[16] Mathur M, Shah H, Dixit K, Khambadkone, Chakrapani A, Irani S. Bacteriological profile of neonatal septicaemia cases (for the year 1990-91). J Postgrad Med 1994; 4-:18-20.

[17] De AS, Rathi MR, Mathur MM. Mortality audit of neonatal sepsis secondary to acinetobacter. J Glob Infect Dis. 2013 Jan;5(1):3-7. doi: 10.4103/0974-777X.107165.

[18] Christian L. Hermansen, Kevin N. Lorah, Respiratory Distress in the Newborn. Am Fam Physician. 2007 Oct 1;76(7):987-994.

[19] Stoll BJ; Hansen NI; Higgins RD; Fanaroff AA; Duara S; Goldberg R, Laptook A; Walsh M; William OH, Ellen RN. Very Low Birth Weight Preterm Infants with Early Onset Neonatal Sepsis: The Predominance of Gram-Negative Infections Continues in the National Institute of Child Health and Human Development Neonatal Research Network, 2002-2003. Pediatric Infectious Disease Journal: July 2005 - Volume 24 - Issue 7 - pp 635-639.

[20] Wai Ho Lim, Yhu-Chering Huang, Ming-Chou Chiang, Ren-Huei Fu, Shih-Ming Chu, Jen-Fu Hsu, Peng-Hong Yang. Prevalence and Pathogen Distribution of Neonatal Sepsis Among Very-Low-Birth-Weight Infants. Pediatrics \& Neonatology. Volume 53, Issue 4 , Pages 228-234, August 2012.

[21] Dankner WM, Spector SA, Fierer J, Davis CE. Malassezia fungemia in neonates and adults: complication of hyperalimentation. Rev Infect Dis. 1987 Jul-Aug; 9(4):743-53.

[22] . Reinhard Berner, Charlotte M Niemeyer, Jekabs U Leititis, Annette Funke, Christiane Schwab, Ute Rau, Kerstin Richter, Moustafa S K Tawfeek1, Andreas Clad and Matthias Brandis. Plasma Levels and Gene Expression of Granulocyte Colony-Stimulating Factor, Tumor Necrosis Factor- $\alpha$, Interleukin (IL)-1 $\beta$, IL-6, IL-8, and Soluble Intercellular Adhesion Molecule-1 in Neonatal Early Onset Sepsis. Pediatric Research 1998;44, 469-477; doi:10.1203/00006450-199810000-00002.

[23] Døllner H, Vatten L, Linnebo I, Zanussi GF, Laerdal A, Austgulen R. Inflammatory mediators in umbilical plasma from neonates who develop early-onset sepsis. Biol Neonate 2001; 80:41-47. 
[24] Krueger M, Nauck MS, Sang S, Hentschel R, Wieland H, Berner R. Cord blood levels of interleukin-6 and interleukin-8 for the immediate diagnosis of early-onset infection in premature infants. Biol Neonate 2001; 80:118-123.

[25] Santana C, Guindeo MC, González G, García-Muñoz F, Saavedra P, Doménech E. Cord blood levels of cytokines as predictors of early neonatal sepsis. Acta Pædiatr 2001; 90:1176-1181.

[26] Tasci Y, Dilbaz B, Uzmez Onal B, Caliskan E, Dilbaz S, Doganci L, et al. The value of cord blood interleukin-6 levels for predicting chorioamnionitis, funisitis and neonatal infection in term premature rupture of membranes. Eur J Obstet Gynecol Reprod Biol 2006; 128:34-39.

[27] Bhat RY, Edward L, Lewis S, Vandana KE. Bacterial isolates of early-onset neonatal sepsis and their antibiotic susceptibility pattern between 1998 and 2004: an audit from a center in India. Italian Journal of Pediatrics 2011; 37:32 doi:10.1186/1824-7288-37-32.

[28] Arani MH, Movahedian A, Arani MG, Adinah M, Mosayebi Z. Predictive Value of Interleukin-6 (IL6) in Term Neonates With Early Sepsis During 2010-2011. Jundishapur J Microbiol. 2013 December; 6(10): e8580. DOI: $10.5812 / \mathrm{jjm} .8580$. 